Introduction Point of care tests (POCTs) for syphilis, HIV, and Trichomonas are currently available, easy-to-use and inexpensive. We surveyed practicing Ob-gyns to determine POCT current use and perceived barriers to use.

Methods Between June and August 2016, 1000 members of the American College of Ob-gyns were randomly selected and invited to complete a Qualtrics survey: 600 were members of the Collaborative Ambulatory Research Network (CARN). Respondents completing at least $60 \%$ of the survey were included in analyses.

Results 930 members had valid emails; 288 (31\%) participated in and completed the survey. $70 \%$ were male. Average years in practice were 16 for males and 23 for females. 30\% reported diagnosing STDs 1-2 times/week and 45\% reported 1-2 times/month. POCTs used included pregnancy test $(83 \%)$, urine dipstick (83\%), wet mount test (79\%) and the vagina $\mathrm{pH}$ test $(54.8 \%)$. Few used Gram stain $(5 \%)$ and stat RPRs (4\%). Newer POCTs were used less frequently with $20 \%$ reporting Affirm VPIII test use, and only 9\% using a rapid HIV test. Most common barriers were the amount of reimbursement received for performing the test (61.9\%) and the payment coverage from the patient (61.3\%).

Conclusion Ob-gyns in the U.S. rely on laboratory test results and older traditional POCTs to diagnosis STDs. Future development and marketing of POCTs should consider not only ease and time of test performance but also cost of tests to the practice and the patient, as well as reimbursement.

\section{P4.15 KNOWLEDGE \& AWARENESS ABOUT STI-HIV AND ITS PREVALENCE AMONG ADOLESCENTS IN INDIA}

Atreyee Sinha, Ankita Siddhanta. International Institute For Population Sciences (IIPS), Mumbai, India

\subsection{6/sextrans-2017-053264.512}

Introduction Sexually transmitted infections and HIV among adolescents in India, has emerged as a separate epidemic in the recent years. STIs elevate the vulnerability of young people to HIV. The present study aimed to evaluate knowledge and awareness of STI-HIV and its prevalence among adolescents in India.

Methods A sample of 20808 unmarried adolescents (15-19 years) from Youth in India: Situation and Needs Study (200607) was analysed. Bi-variate and multivariate techniques were applied to accomplish the objective.

Results Although $80 \%$ of the adolescents knew about HIV/ AIDS, only $11 \%$ had heard about STIs. Only half of the respondents had comprehensive knowledge on the two ways of HIV prevention (condom use and single partner relation). While boys were more aware about STIs and HIV/AIDS, girls lagged behind in having accurate information. Nearly one third of the adolescents had common misconceptions about HIV transmission. STI-HIV awareness was more among adolescents with higher education, living in urban areas, belonging to rich families and those who received sex education. Around $10 \%$ of the adolescents - $4 \%$ boys and $12 \%$ girls, reported symptoms of STI in past 3 months. STIs were more prevalent among illiterates, living in rural areas, belonging to poor and middle class families and who received sex education from proper source like family, media and $\mathrm{NGO} /$ health workers. Logistic regression revealed that adolescent girls $(p<0.001)$ and respondents from rural areas $(\mathrm{p}<0.01)$ were significantly more likely to report STI symptoms. Respondents who received sex and family life education from reliable sources were more likely to report STI problems. Adolescents belonging to upper wealth quintile were $24 \%$ less likely $(\mathrm{p}<0.01)$ and who had high awareness of STI-HIV were 12\%-15\% less likely to report any symptom $(\mathrm{p}<0.10)$.

Conclusion Findings underscore limited awareness about STIs, HIV prevention and transmission specifically among adolescent girls. There is an immense need to implement gender-based sex education in schools and social campaigning to increase awareness.

\section{P4.16 THE IMPACT OF POINT-OF-CARE MANAGEMENT ON THE TRANSMISSION OF ANOGENITAL GONOCOCCAL INFECTIONS AMONG MEN WHO HAVE SEX WITH MEN IN AMSTERDAM: A MATHEMATICAL MODELLING AND COST-EFFECTIVENESS STUDY}

${ }^{1} \mathrm{M}$ Bartelsman, ${ }^{2} \mathrm{~K}$ Vaughan, ${ }^{1}$ Van Rooijen Ms, ${ }^{1}$ De Vries Hjc, ${ }^{3} \mathrm{M}$ Xiridou. ${ }^{1}$ STI Outpatient Clinic, Public Health Service of Amsterdam, Amsterdam, The Netherlands; ${ }^{2}$ Bang for Buck Consulting, Amsterdam, The Netherlands; ${ }^{3}$ National Institute for Public Health and The Environment (R/VM), Bilthoven, The Netherlands

\subsection{6/sextrans-2017-053264.513}

Introduction Point-of-care (POC) management (providing test results during the initial visit) may be more effective as testing policy than conventional testing (providing test results after days) by averting transmissions occurring between testing and treatment or due to loss to follow-up. We investigated the impact of POC management (with light microscopic examination of Gram stained smears) of anogenital gonorrhoea among men who have sex with men (MSM) on gonorrhoea prevalence and testing and treatment costs at the STI clinic of Amsterdam.

Methods Data on costs and sexual behaviour of MSM were collected at our clinic. With a deterministic model we described gonorrhoea transmission among MSM in Amsterdam; we calculated the prevalence of gonorrhoea, testing and treatment costs and the numbers of consultations over five years in our clinic, in three testing scenarios: POC for symptomatic MSM only (the current scenario), POC for all MSM, and no POC for MSM.

Results Among MSM, 34.7\% (109/314) had sexual contacts in the period between testing and treatment (average of 13 days), of whom 22.9\% (25/109) had unprotected anal intercourse. Expanding POC testing of symptomatic MSM to all MSM resulted in a 5 year decline in gonorrhoea prevalence of $10.7 \%$ (IQR, $8.2 \%-15.4 \%$ ) at an increase of $€ 2.40(8.6 \%)$ per consultation and $€ 86118$ overall (+8.3\%). Switching from POC testing of symptomatic MSM to no POC testing increased prevalence by $59.9 \%$ (IQR, 25.6\%-126.7\%) with cost savings of $6.5 \%(€ 1.83)$ per consultation and $€ 54044$ $(-5.2 \%)$ in total costs after 5 years.

Conclusion The abandonment of POC gonorrhoea testing of symptomatic MSM would result in a high increase in gonorrhoea prevalence against a reduction in costs per consultation, while expanding to all MSM would result in a modest decrease in prevalence against a rise in costs per consultation. While the outcomes depend on specific local characteristics, such as the prevalence, loss to follow up, and sexual behaviour, the developed framework of this study can be useful to evaluate POC management in other settings. 